\title{
El cambio climático como riesgo y amenaza para la seguridad: derivaciones en el desarrollo del régimen jurídico internacional en materia de clima
}

\section{Climate Change as Risk and Security Threat: Implications for the Evolution of the International Climate Change Regime}

\author{
Rosa Giles Carnero ${ }^{1}$ \\ Universidad de Huelva (España)
}

Recibido: 16-01-16

Aprobado: 22-03-16

\section{Resumen}

La interconexión entre el fenómeno del cambio climático y las diferentes aproximaciones al concepto de seguridad es amplia y presenta aspectos muy diversos. Con este presupuesto inicial, el presente estudio pretende analizar las posibles derivaciones de esta interconexión en el desarrollo de la acción jurídica internacional en materia de cambio climático. El régimen jurídico internacional en materia de clima se ha articulado como el principal foro en el que abordar una respuesta global al cambio climático, de forma que resulta interesante reflexionar sobre el impacto que puede tener una aproximación basada en la seguridad tanto en el progreso general del régimen, como en el diseño de algunos de sus elementos jurídicos concretos.

Palabras-clave: Cambio Climático, Seguridad Climática, Proceso de Securitización.

\footnotetext{
${ }^{1}$ (giles@uhu.es) Profesora Titular de Derecho Internacional Público, Universidad de Huelva. El presente estudio se enmarca dentro del proyecto de investigación: "Las Respuestas del Derecho Internacional y Europeo a los Nuevos Riesgos y Amenazas Contra la Seguridad Humana" (RASEGUR), Plan Nacional de I+D+I (Ref.: DER2015-65906-P) y de la Red de Excelencia sobre "Los actuales desafíos del Derecho Internacional", del Plan Estatal de Investigación Científica y Técnica y de Innovación 2013-2016 (DER15-69273-RED).
} 


\begin{abstract}
The interconnection between climate change and security is broad and complex. Taking this framework into account, this study aims to analyse the implications of this interconnection for the development of the international climate action. The international climate change regime is the main discussion forum for adopting effective climate strategies, and a security approach may be useful for the progress of the regime, and the design of new rules and norms.
\end{abstract}

Key-words: Climate Change, Climate Security, Securitization Process.

\title{
1. Introducción.
}

La literatura académica que puede encontrarse sobre las implicaciones para la seguridad del fenómeno del cambio climático es muy abundante, de forma que se ha abordado el análisis de la interconexión entre este fenómeno ambiental y la generación de situaciones de riesgo y conflicto desde muy diversas aproximaciones. La investigación en el ámbito de la seguridad ha ampliado su campo de análisis para incluir la reflexión sobre la seguridad ambiental y energética, y en este escenario el fenómeno del cambio climático aparece como una amenaza que será objeto de una especial atención. Estos estudios aportan una aproximación diferente a la que suele desarrollarse desde el campo de la investigación de carácter ambientalista, transformando conceptualmente el riesgo ambiental que supone las modificaciones climáticas en una amenaza para la seguridad y aportando, de esta forma, un elemento de reflexión complementario sobre la actuación internacional que debe desarrollarse para la mitigación y la adaptación al cambio climático.

Las posibilidades a la hora de abordar el análisis de la interconexión entre cambio climático y seguridad son, por lo tanto, muy amplias y diversas, y entre ellas el presente estudio pretende desarrollar un acercamiento en el que el principal objetivo sea apuntar las posibles derivaciones que para el desarrollo de la acción jurídica internacional en materia de cambio climático puede tener esta interacción. El régimen jurídico internacional en materia de clima se reconoce en la escena internacional como el principal foro en el que abordar una respuesta global al cambio climático que evite sus consecuencias más catastróficas, y diseñe soluciones de adaptación a los efectos ya producidos o inminentes. Por lo tanto, la acción internacional en el ámbito climático va a diseñarse y desarrollarse prioritariamente en este espacio, y es por ello que resulta interesante reflexionar sobre el impacto que puede tener una aproximación basada en la seguridad tanto en el progreso general del régimen, como en el diseño de algunos de sus elementos jurídicos concretos. 
En esta reflexión no puede olvidarse que el régimen internacional en materia de clima se encuentra en un momento crucial de su desarrollo, lo que puede hacer más interesante, si cabe, la reflexión sobre las implicaciones que los cambios climáticos presentan en materia de seguridad y que van más allá de las netamente ambientales. El estado actual de la acción jurídica internacional en materia de cambio climático hace particularmente relevante el análisis de la capacidad que pueda tener una perspectiva basada en la seguridad, para el impulso adicional en la adopción de compromisos climáticos de los Estados Partes. En los últimos años el régimen internacional en materia de clima ha sufrido una importante paralización, debido a la reticencia de los Estados, en particular de los grandes emisores de gases de efecto invernadero, a asumir compromisos relevantes para la mitigación y la adaptación al cambio climático, es en este contexto en el que aquellas aproximaciones que puedan aportar elementos de solución a este desbloqueo y reflexiones sobre técnicas eficaces de actuación, aparecen como especialmente relevantes y útiles.

A lo apuntado debe añadirse que cualquier análisis que se realice teniendo como objeto la interacción entre cambio climático y seguridad, ha de tener en cuenta el desarrollo que el concepto de seguridad ha experimentado desde el final de la Guerra Fría. La evolución de esta noción ha conllevado una ampliación en los ámbitos de estudio, de forma que a la reflexión sobre las amenazas clásicas a la seguridad del Estado se ha unido la que pone el énfasis en la de individuos y colectividades ${ }^{2}$.El presente trabajo parte tanto de los elementos que conforman el momento actual del régimen internacional en materia de clima, como del reconocimiento de que a las nociones conceptuales clásicas de la seguridad se han sumado otras novedosas que amplían el concepto y el campo de estudio en este ámbito. De forma breve y sin pretender desarrollar un análisis exhaustivo, en este apartado introductorio merece la pena detenerse en algunas consideraciones relacionados con el momento actual del régimen internacional en materia de clima, y con el desarrollo del concepto de seguridad, ambas cuestiones analizadas con el objetivo de contextualizar adecuadamente el estudio realizado.

Como se ha apuntado, la acción internacional en materia de cambio climático se ha desplegado prioritariamente en el marco del régimen jurídico internacional en materia de clima incluido en el sistema de Naciones Unidas. Este régimen se ha estructurado conforme a las previsiones de la Convención Marco de las Naciones Unidas para el Cambio Climático (CMNUCC) de 9 de mayo de 1992, y desarrollado mediante el Protocolo de Kioto de 11 de

\footnotetext{
${ }^{2}$ Para un mayor análisis de la evolución y ampliación de los campos de estudio definidos desde el análisis de la seguridad, con un apartado específico dedicado a la seguridad ambiental, ver la obra de María Isabel Torres Cazorla y Elena del Mar García Rico, coordinadoras, La seguridad internacional en el siglo XXI: nuevas perspectivas, Plaza y Valdés Editores, 2011.
} 
diciembre de $1997^{3}$. Sobre la base de estos dos tratados internacionales, se ha articulado la estructura jurídica y orgánica en la que se sostiene y desarrolla la gestión internacional en materia de cambio climático, componiéndose un sistema evolutivo en el que diseñar una estrategia global y continuada frente a esta amenaza ambiental ${ }^{4}$.

No cabe duda de que el régimen internacional en materia de cambio climático ha desarrollado un sistema en el que se han ensayado novedades jurídicas y de gestión interesantes para la evolución no sólo de la acción climática, sino de todo un sector internacional como es el Derecho Internacional del Medio Ambiente. No obstante y pese a esta afirmación, no puede soslayarse la fundada crítica de su escaso impacto para la resolución del problema ambiental que enfrenta. La reticencia de los Estados a adoptar medidas costosas para la mitigación del cambio climático tiene como efecto que no pueda asegurarse el objetivo de evitar un calentamiento global del planeta con repercusiones potencialmente catastróficas, y por lo tanto pone en duda la eficacia de todo un complejo sistema jurídico y orgánico que pretende seguir avanzando para superar esta situación.

El momento actual del régimen internacional en materia de clima es el de una fase transitoria hasta 2020, en la que se desarrolla el segundo periodo de cumplimiento del Protocolo de Kioto al tiempo que se ha adoptado el pasado mes de diciembre de 2015 un nuevo acuerdo que podría entrar en vigor a partir de aquella fecha. El Protocolo de Kioto de 1997 se diseñó mediante una primera fase de cumplimiento que culminó en 2012, y que debía continuarse posteriormente. La segunda fase de cumplimiento abarca desde 2013 a 2020, y se ha materializado a través de la adopción de la Enmienda de Doha pendiente de entrar en vigor. No obstante, el limitado éxito ambiental de la puesta en marcha del Protocolo de Kioto conllevó que se exploraran nuevas vías en la negociación internacional, que apostaron por el diseño de un nuevo "protocolo,

\footnotetext{
${ }^{3}$ Convención marco de las Naciones Unidas sobre el cambio climático, hecha en Nueva York (Estados Unidos) el 9 de mayo de 1992, y con entrada en vigor el 21 de marzo de 1994. Naciones Unidas, Treaty Series, vol. 1771, p. 107, disponible en: http://unfccc.int/essential_background/ convention/items/6036.php (última consulta el 15 de enero de 2016); y Protocolo de Kioto a la Convención marco de las Naciones Unidas sobre el cambio climático, adoptado en Kioto (Japón) el 11 de diciembre de 1997, y con entrada en vigor el 16 de febrero de 2005. Naciones Unidas, Treaty Series, vol. 2303, p. 162, disponible en: http://unfccc.int/kyoto_protocol/items/2830.php(última consulta el 15 de enero de 2016).

La CMNUCC cuenta en la actualidad con la participación de 196 Estados Partes, lo que asegura su carácter de foro de negociación global; la participación en el Protocolo de Kioto se ha limitado a 192 Partes, destacando entre las ausencias a Estados Unidos que no llegó a ratificar este instrumento pese a haber participado activamente en su preparación; y Canadá, único Estado que se ha retirado del Protocolo de Kioto siguiendo el procedimiento de denuncia establecido en su artículo 27.1.

${ }^{4}$ Para un mayor análisis del régimen en materia de clima como modelo de gestión de un riesgo ambiental global, ver Rosa Giles Carnero, El Protocolo de Kioto como modelo de gestión ambiental global, en [Antonio Remiro Brotóns y Rosa M $\mathrm{M}^{\mathrm{a}}$ Fernández Egea, eds., El Cambio Climático en el Derecho Internacional y Comunitario, Fundación BBVA, 2009], pp. 27-601.
} 
otro instrumento jurídico o una conclusión acordada con fuerza legal" en el marco de la $\mathrm{CMNUCC}^{5}$. Como culminación de este proceso negociador desarrollado en los últimos años, se ha diseñado un nuevo texto internacional mediante el que desarrollar nuevos compromisos de mitigación y adaptación al cambio climático durante la pasada Conferencia de las Partes de la CMNUCC, celebrada en París del 30 de noviembre al 11 de diciembre de $2015^{6}$.

El Acuerdo de París se abrirá a la firma en abril de 2016, y a partir de entonces se iniciará el proceso de ratificaciones que debería permitir su entrada en vigor al finalizar el año 2020. El nuevo acuerdo incluye un nuevo enfoque en la gestión del cambio climático, ya que abandona el modelo del Protocolo de Kioto, en el que se fijaban obligaciones concretas de reducción de gases de efecto invernadero de los Estados desarrollados, para establecer un sistema basado en las llamadas Contribuciones Previstas Determinadas a Nivel Nacional (INDCs, por sus siglas en inglés) mediante las que el compromiso de cada Estado se conforma tomando como base la declaración de las medidas que tiene previsto adoptar y utilizando unas metodologías homogéneas ${ }^{7}$. La deriva y efectividad del Acuerdo de París es aún incierta, por lo que aunque no cabe duda de que supone el principal elemento de avance del régimen en materia de cambio climático, no puede olvidarse que en el momento actual el texto principal de referencia para la gestión global de este fenómeno ambiental sigue siendo la CMNUCC, y con éste el sistema jurídico y orgánico establecido conforme a sus previsiones.

El esquema apuntado de la evolución del régimen en materia de clima muestra lo crucial de desarrollar la capacidad de generar compromisos por parte de los Estados, y es en este punto en el que la conexión de este fenómeno con el concepto de seguridad puede tener como consecuencia nuevos enfoques en algunos elementos, así como un nuevo impulso. No obstante, la primera cuestión que surge a la hora de reflexionar sobre este posible impacto, es la de delimitar el concepto de seguridad que debe utilizarse. Ya se ha apuntado que desde el final de la Guerra Fría el concepto de seguridad ha experimentado una importante extensión, para incluir junto a las amenazas tradicionales

\footnotetext{
${ }^{5}$ Ver Decisión 1/CP.17, Establecimiento de un Grupo de Trabajo Especial sobre la Plataforma de Durban para una Acción Reforzada, de la Conferencia de las Partes de la CMNUCC, par. 2, disponible en: http://unfccc.int/meetings/durban_nov_2011/meeting/6245/php/view/decisions.php (última consulta el 15 de enero de 2015).

Para un mayor análisis de la articulación jurídica de esta fase transitoria, ver Rosa Giles Carnero, La acción internacional y europea en materia de cambio climático: construyendo la nueva fase 20132020 [en Isabel Rodríguez Martínez, directora, La negociación de emisiones GEI en los mercados de carbono. Régimen y regulación, Thomson Reuters Aranzadi, 2014], pp. 39-62.

${ }^{6}$ Ver Decisión 1/CP.21, Adopción del Acuerdo de París, de la Conferencia de las Partes de la CMNUCC (en la fecha de cierre del presente trabajo, 15 de enero de 2016, la numeración de las Decisiones de la $21^{\circ}$ sesión de la Conferencia de las Partes es provisional), disponible en: http:// unfccc.int/2860.php (última consulta el 15 de enero de 2016).

${ }^{7}$ Ver art. 3 y siguientes, Decisión 1/CP.21, Adopción del Acuerdo de París.
} 
a la integridad territorial del Estado otras de carácter más difuso como las económicas, sociológicas o ambientales. Este proceso ha llevado a que junto a los términos tradicionales de seguridad nacional o internacional, aparezcan otros como el de seguridad humana, mediante el que se intenta poner el énfasis en la necesidad de protección del ser humano más allá de la del Estado.

No es el objetivo de este estudio abordar el complejo y amplio análisis de la extensión del concepto de seguridad, pese a lo cual se ha optado por utilizar los tres términos señalados de forma que sea posible abordar diversos aspectos de la interrelación entre cambio climático y seguridad que inciden o pueden incidir en el desarrollo del régimen jurídico internacional en materia de clima. El estudio que sigue trata de analizar el impacto que ha tenido y que puede desarrollar la interconexión de los diferentes aspectos del amplio concepto de seguridad sobre el desarrollo de la gestión global del cambio climático, en un examen que necesariamente no puede ser exhaustivo, ni por razón de materia ni de espacio, pero que aspira a presentar algunos de los problemas y las potencialidades que esta interconexión presenta. No cabe duda de que las agendas de la seguridad y la del cambio climático han sufrido una interconexión debido a la amplitud de los posibles efectos de este fenómeno ambiental y a la extensión del concepto de seguridad, la cuestión es si esta interconexión puede aportar un nuevo enfoque en la toma de decisión, en la implementación de medidas, y sobre todo en la generación de compromisos climáticos internacionales que permita avanzar en la efectividad del régimen internacional en materia de clima.

Conforme a lo señalado, en las páginas siguientes se incluye el análisis del cambio climático utilizando una aproximación basada en las nociones de seguridad nacional, internacional y humana sucesivamente. Aunque algunos elementos de análisis que se exponen en los diferentes apartados son comunes a los tres, esta diferenciación permite el estudio ordenado de las diferentes derivaciones de una aproximación basada en la seguridad en el desarrollo del régimen en materia de clima. Como se ha señalado, no se pretende en modo alguno un análisis profundo de los conceptos de seguridad nacional, internacional y humana, sino una aproximación con una estructura de análisis que resulte útil para conformar un tapiz en el que abordar la relación entre cambio climático y seguridad desde diversas ópticas, ya que todas ellas suponen aportar un punto complementario de reflexión sobre la gestión internacional de este reto ambiental. Se finalizará con un apartado de conclusiones, en el que se incluyen algunas consideraciones sobre la valoración general de la incidencia que una aproximación basada en la seguridad ha tenido, y puede llegar a tener, en el régimen internacional en materia de clima. 


\section{El cambio climático desde la perspectiva de la seguridad nacional}

Como ya ha sido apuntado en el apartado introductorio, desde el final de la Guerra Fría diversos ámbitos de la práctica nacional e internacional tradicionalmente considerados fuera del ámbito de la seguridad se han integrado en éste, siguiendo lo que se ha denominado comúnmente como un "proceso de securitización". En este proceso un asunto que no se percibía como amenaza pasa a conceptualizarse de esta forma, y se sitúa como un nuevo desafío para la seguridad. Los análisis académicos sobre cómo se produce este proceso y sus efectos son abundantes en la doctrina, como también han sido abundantes las críticas que ha suscitado y que inciden prioritariamente en el hecho de que este proceso puede conllevar la eliminación de garantías a la hora de la toma de decisiones $^{8}$. Los procesos de securitización en los sistemas nacionales suponen incluir un determinado ámbito en la agenda de la seguridad, y esto hace posible que puedan adoptarse medidas excepcionales al margen de los sistemas de control habituales de la toma de decisión. Esta posibilidad es la que ha activado el principal núcleo de sospecha y crítica respecto a los efectos últimos de los procesos de securitización en los sistemas nacionales, y ensombrece otras supuestas utilidades que pudieran argumentarse.

En lo que respecta al cambio climático, en las últimas décadas la preocupación por este fenómeno ambiental se ha introducido en las agendas de seguridad nacional de la mayoría de los Estados occidentales, de forma que puede afirmarse que se ha producido un claro proceso de securitización en este ámbito y en este tipo de Estados. Esta incorporación a las agendas de seguridad se ha realizado incluyendo diversos términos y conceptos, entre los que destacan los de riesgo, amenaza o factor multiplicador de otras amenazas a la seguridad. En todos los casos la idea de base es que los efectos del cambio climático pueden provocar perturbaciones en la seguridad nacional, aunque resulta complicado poder establecer una terminología y una caracterización que puedan considerarse generales en las diferentes definiciones que se utilizan en las estrategias de seguridad nacional de los diferentes Estados ${ }^{9}$.

\footnotetext{
${ }^{8}$ En el campo del análisis de las relaciones internacionales destaca el amplio trabajo sobre la teoría de la securitización desarrollada por la Escuela de Copenhague de Estudios de Seguridad, en el que se registra un esfuerzo de reflexión sobre los diferentes elementos que componen el proceso de securitización. Pueden consultarse algunos resultados de sus trabajos en Barry Buzan, Ole Wæver y Jaap de Wilde, Security. A New Framework for Analysis, Lynne Rienner Publishers, 1998. $\mathrm{La}$ influencia de estos trabajos puede verse de forma amplia en la doctrina, como se constata en publicaciones referidas específicamente al cambio climático como Günter Brauch, Peter Michael Link y Janpeter Schilling, eds., Climate Change, Human Security and Violent Conflict. Challenges for Societal Stability, Springer, 2012; aunque también se ha señalado que, después de un amplio debate con críticas y contra-críticas, se está lejos de poder afirmar que exista una teoría unificada y completa sobre securitización, ver Thierry Balzacq, Securitization Theory: How Security Problems Emerge and Dissolve, Routledge, 2010.

${ }^{9}$ Los términos riesgo y amenaza han tratado de definirse en la doctrina, aunque en muchos casos 
Cabe destacar que la acción del gobierno británico es la que puede considerarse como pionera a la hora de iniciar el proceso de securitización del cambio climático, ya que desde hace más de una década introdujo este fenómeno ambiental en su agenda de seguridad e impulsó que se abordara en el marco del Consejo de Seguridad de Naciones Unidas ${ }^{10}$. Los diferentes socios europeos han transitado también por este proceso, y el caso español es ya un ejemplo más en este desarrollo. La Estrategia de Seguridad Nacional: Un proyecto compartido, de mayo de 2013, se ocupa del cambio climático, aunque en este texto no se califica directamente como riesgo o amenaza. El Tercer Capítulo de la Estrategia lleva por título "Los riesgos y amenazas para la Seguridad Nacional", pero en su texto incluye no sólo estas categorías, sino también los denominados "factores potenciadores" definidos como aquellos "que pueden generar nuevos riesgos o amenazas o multiplicar y agravar sus efectos". Es en este último grupo en el que se incluye al cambio climático ${ }^{11}$. Con esta fórmula se sigue una tendencia presente también en la escena internacional, en la que se incluye con plena claridad al cambio climático en la agenda de la seguridad pero sin definir a un fenómeno ambiental como una amenaza a la seguridad de forma autónoma, sino destacando sus efectos sobre otros riesgos y amenazas.

También Estados Unidos ha incluido la cuestión climática en su agenda de seguridad y, particularmente, resulta interesante las referencias a este ámbito en su Estrategia de Seguridad Nacional de febrero de 2015, la segunda del Presidente Barak Obama. En este texto se alude al cambio climático tanto como una amenaza, como un multiplicador de otras ${ }^{12}$. Las dos posibles caracterizaciones del cambio climático como una preocupación de la

con muy diversos resultados en función de la aproximación que se utilizara en cada estudio. Quizás lo más relevante en este ámbito es la calificación de riesgo cuando esto supone una ampliación de la percepción de la seguridad. Así, por ejemplo, Gracia Abad Quintanal define amenazas como "posibles acciones orientadas a infligir un daño que siempre son de carácter intencionado", mientras que riesgos son caracterizados como "posibles situaciones que podrían darse como consecuencia de un conjunto de circunstancias y que conllevarían un daño en caso de producirse", son los segundos lo que la autora considera que más proliferan en los nuevos desafíos de la seguridad, de forma que también se introduce un alto grado de incertidumbre en el concepto de seguridad. Ver El concepto de seguridad: su transformación, "Comillas Journal of International Relations", 4 (2015), pp. 41-51, p. 43.

${ }^{10}$ Sobre la actividad británica en el proceso de securitización tanto nacional como internacional, puede verse Katie Harris, Climate Change in UK Security Policy: Implications for development Assistance?, Working Paper 342. Overseas Development Institute, 2012.

${ }^{11}$ Entre los factores potenciadores la Estrategia va a detenerse en el cambio climático, y lo define como "el gran desafío ambiental y socioeconómico del S. XXI" que plantea grandes retos para la seguridad porque puede convertirse en un multiplicador de amenazas. Ver Estrategia de Seguridad Nacional: Un proyecto compartido, de mayo de 2013, p. 21, disponible en: http://www.lamoncloa. gob.es/documents/seguridad_1406connavegacionfinalaccesiblebpdf.pdf (última consulta el 15 de enero de 2016).

${ }^{12}$ En palabras de la Estrategia: "climate change is an urgent and growing threat to our national security, contributing to increase natural disasters, refugee flows, and conflicts over basic resources like food and water". Ver National Security Strategy, febrero 2015, p. 12, disponible en: https://www. whitehouse.gov/sites/default/files/docs/2015_national_security_strategy.pdf (última consulta el $15 \mathrm{de}$ enero de 2016). 
seguridad son incluidas de esta forma, destacándose así la complejidad que este fenómeno puede tener para la seguridad al conllevar una serie de efectos desestabilizadores tanto intrínsecos como en relación a otros factores. En la Estrategia estadounidense destaca además una aproximación que va a ser de especial interés en la cuestión que nos ocupa en el presente trabajo, ya que a lo largo de todo el texto se señala la importancia del liderazgo de Estados Unidos para enfrentar las diversas amenazas que identifica ${ }^{13}$. Este enfoque va a ser particularmente importante en el desarrollo del régimen internacional en materia de clima, ya que la decisión de liderazgo de Estados Unidos supone una oportunidad clara de avance como se verá más adelante en este apartado, e incluir esta materia en la agenda de seguridad puede resultar conveniente para soslayar la resistencia interna a desarrollarlo.

Los ejemplos expuestos muestran una práctica de los Estados desarrollados en la que el cambio climático ha pasado a formar parte de las agendas de seguridad. En lo que interesa a este estudio, la pregunta clave es si este proceso de securitización puede influir en la posición internacional que desarrollen en el marco del régimen internacional en materia de clima y, en particular, si puede favorecer la aceptación de compromisos internacionales de mitigación y adaptación al cambio climático. Lo que se está planteando es la cuestión de si es más fácil para los Estados adoptar decisiones costosas cuando hay una percepción de amenaza a la seguridad, y si el miedo a posibles efectos catastróficos en este ámbito puede ser un buen aliado para la actuación ambiental.

En el desarrollo del Derecho Internacional del Medio Ambiente puede apreciarse que la percepción de un riesgo importante es un buen acicate para la toma de decisiones, pero en el ámbito particular del cambio climático debe recordarse que persiste un alto grado de incertidumbre respecto a sus consecuencias concretas, por lo que esa percepción de miedo a sus efectos potencialmente catastróficos ha sido difícil de desarrollar y mantener. Es en este escenario particular donde el temor a efectos no únicamente ambientales, sino también relacionados con la seguridad, puede tener un mayor protagonismo a la hora de promover el apoyo a la adopción de decisiones con un alto coste económico inmediato. De forma general puede señalarse, por tanto, que el apoyo en el sistema nacional a la aceptación de compromisos internacionales puede verse favorecido al establecerse el énfasis en la conexión entre cambio

\footnotetext{
${ }^{13} \mathrm{Al}$ analizar la Estrategia, Ignacio José García Sánchez va a destacar que "la nueva ESN se esfuerza por reafirmar la centralidad y oportunidad del liderazgo estadounidense como nación indispensable frente a las principales amenazas que se ciernen sobre la humanidad", ver La Estrategia de Seguridad Nacional de los Estados Unidos de América 2015, "Documento de Análisis. Instituto Español de Estudios Estratégicos" 9 (2015), pp. 1-9, p. 5. Este liderazgo se afirma en clima en el texto de la propia Estrategia: "these complex times have made clear the power and centrality of America's indispensable leadership in the world. We mobilized and are leading global efforts to [...] turn the corner on global carbon emissions", op. cit., p. 2.
} 
climático y seguridad nacional. Desde esta óptica, el proceso de securitización puede resultar favorable para superar uno de los mayores escollos de la acción internacional en materia de cambio climático como es la falta de voluntad de los Estados para asumir compromisos concretos.

Aunque la conformación de la posición de los Estados ha resultado siempre un elemento clave para el desarrollo del régimen en materia de cambio climático, y explica en buena medida la paralización sufrida en los últimos años en la adopción de compromisos más ambiciosos, en el momento actual este protagonismo es mayor si cabe. En la fase presente y para la articulación de compromisos concretos de mitigación y adaptación al cambio climático, los Estados deben prestar su consentimiento para que entren en vigor tanto la Enmienda de Doha, que incluye el segundo periodo de cumplimiento del Protocolo de Kioto hasta 2020, como el Acuerdo de París que debería entrar en vigor después de esa fecha. La prestación del consentimiento a estos dos textos es lo que asegura la continuidad, y posible eficacia, del régimen internacional en materia de cambio climático. En el momento actual, por tanto, la expresión de la voluntad de los Estados a vincularse por los textos adoptados en el marco de la Conferencia de las Partes resulta fundamental para la evolución del sistema.

Cabe, no obstante, señalar un elemento más que caracteriza las nuevas medidas adoptadas en el seno de la Conferencia de las Partes, y que otorga aún más protagonismo a la voluntad y la actividad nacional en el desarrollo del régimen internacional. Ya se ha apuntado en el apartado introductorio que la pieza clave del Acuerdo de París son las INDCs, y la consecuencia inmediata de esto es que la labor desarrollada en los sistemas nacionales aparece como determinante en la conformación misma de la obligación internacional.

El sistema de compromisos incluido en el Acuerdo de París huye del adoptado en Kioto, en el que se establecían unas obligaciones específicas de reducción de emisiones para los Estados Partes desarrollados, que debían ser cumplidas en unos periodos determinados. El compromiso concreto se fijaba, por tanto, en el ámbito internacional, y quedaba perfectamente delimitado en este nivel. Un nuevo acuerdo internacional que siguiese este modelo parecía abocado al fracaso, ya que diversos Estados habían mostrado su negativa a asumir obligaciones concretas fijadas en el sistema internacional y, entre ellos, los principales emisores como son Estados Unidos y China. Para tratar de superar esta negativa, el nuevo modelo diseñado en el Acuerdo de París se basa en la recepción y la revisión de las INDCs, de forma que el compromiso climático se desarrolle mediante la colaboración entre los sistemas nacionales e internacional. Son los Estados los que comunican las acciones que están dispuestos a asumir en materia de mitigación y adaptación al cambio climático, de forma que el sistema internacional procederá a revisarlas de acuerdo con metodología comunes, y hacer un seguimiento que permita incrementar 
progresivamente la ambición de los resultados asumidos. Puede observarse, por tanto, que si en cualquier tratado internacional la actuación de los sistemas nacionales es clave para su correcta implementación, en este caso esta actuación pasa a serlo también en la conformación misma de la obligación internacional. En este contexto, el compromiso de los Estados con una acción eficaz en materia climática resulta la pieza clave de un sistema internacional que se diseña como un método general de verificación, homologación y facilitación de los esfuerzos nacionales en este ámbito.

La situación y el diseño actual del régimen internacional en materia de clima promueven que se ponga el énfasis en aquellos elementos que puedan promover la conformación de una voluntad nacional favorable a asumir compromisos climáticos, tanto respecto a la ratificación de los textos adoptados como en relación al contenido de las INDCs, y en este ámbito es donde el proceso de securitización en los sistemas nacionales puede aparecer con efectos favorables. Ahora bien, no puede olvidarse que estos procesos en relación al cambio climático se han producido prioritariamente en los Estados desarrollados, de forma que los Estados en vías de desarrollo se resisten a incluir el fenómeno del cambio climático en el concepto de seguridad nacional, y prefieren vincularlo al de desarrollo sostenible. Esta postura se conecta con un llamamiento claro a la necesidad de financiación que estos Estados van a reclamar para la implementación de medidas de mitigación y adaptación al cambio climático, y en todo caso y como veremos en otros apartados parecen más proclives a la utilización de conceptos amplios de seguridad que puedan vincularse a la necesidad de desarrollo.

Conforme a lo señalado, puede afirmarse que dado que el proceso de securitización se ha producido prioritariamente en sociedades occidentales desarrolladas, es en estos escenarios en los que debe contextualizarse el impacto que puede tener en la evolución de la posición internacional de estos Estados ${ }^{14}$. Esta precisión no resta importancia a esta aproximación, ya que se está circunscribiendo precisamente a los Estados más desarrollados y, por lo tanto, incluidos en el listado de principales emisores de gases de efecto invernadero, pero sobre todo, esta precisión conduce a considerar que esta aproximación va a ser interesante sobre todo para valorar la posición de un Estado en particular: Estados Unidos ${ }^{15}$.

${ }^{14}$ Resulta pertinente recordar en este ámbito las críticas recibidas por la Escuela de Copenhague de Estudios de Seguridad a la hora de desarrollar una teoría global de securitización. De forma reiterada se ha señalado que esta teoría asume un modo de entender la sociedad eurocéntrico, que pretende exportarse con un éxito incierto. Ver Claire Wilkinson, The Copenhagen School on Tour in Kyrgyzstan: Is Securitization Theory Useable Outside Europe?, "Security Dialogue", (38) 2007, pp. $5-25$.

${ }^{15}$ En los discursos inaugurales de los líderes en el inicio de la $21^{\mathrm{a}}$ sesión de la Conferencia de las Partes de la CMNUCC el 30 de noviembre de 2015 pudo apreciarse con claridad las alusiones referidas a la seguridad que se formulaban por los líderes de los Estados desarrollados. Tanto el Presidente de 
La relevancia de la participación activa de Estados Unidas para la eficacia del régimen internacional en materia de clima deviene no sólo de que es el segundo emisor global de gases de efecto invernadero, sino también de su demostrada capacidad de liderazgo en los regímenes ambientales. La negativa estadounidense a ratificar el Protocolo de Kioto es uno de los elementos significativos que han propiciado su paralización, y esta conclusión aparece con más claridad si cabe al observarse que el Acuerdo de París ha sido fruto, en buena medida, de la diplomacia estadounidense de nuevo implicada en el liderazgo climático. En este contexto, no pocos analistas señalan la conveniencia del proceso de securitización del cambio climático en el sistema estadounidense para facilitar un giro hacia posiciones más ambiciosas respecto a la aceptación de compromisos internacionales de mitigación de cambio climático, y apuntan a este proceso como una de las claves que ha permitido una posición más ambiciosa de Estados Unidos en el proceso de negociación del nuevo Acuerdo de París ${ }^{16}$.

El compromiso de Estados Unidos con el régimen de cambio climático es crucial para su eficacia, tanto en el ámbito de la definitiva ratificación del Acuerdo de París, como en el desarrollo de una INDC ambiciosa. Para el desarrollo de este compromiso, Estados Unidos tendrá que superar una férrea resistencia desde diferentes sectores domésticos, y es en este ámbito en el que la configuración del cambio climático como una amenaza a la seguridad nacional aparece como un elemento definitorio en la toma de decisión. Desde esta perspectiva, el proceso de securitización del cambio climático en Estados Unidos se ha mostrado como un elemento que ha favorecido su compromiso y liderazgo en el régimen internacional en materia de cambio climático, y se ha convertido, por tanto, en unos de los elementos que han impulsado la adopción de un nuevo instrumento para el desarrollo de medidas de mitigación y adaptación al cambio climático.

los Estados Unidos, Barak Obama, como el Presidente de la Comisión Europea, Jean-Claude Juncker, destacaron la importancia de tomar medidas para evitar los riesgos para la seguridad. El ambiente de la Conferencia de las Partes estaba en aquellos momentos enrarecido por la preocupación también de seguridad de una amenaza muy dispar como era la del terrorismo, pero en estos discursos inaugurales no faltaron los recordatorios de la interconexión entre el fenómeno del cambio climático y la seguridad nacional para afianzar el compromiso ambiental de buena parte de los Estados desarrollados. Pueden consultarse los discursos inaugurales en: http://unfccc.int/meetings/paris_nov_2015/items/9331.php (última consulta el 15 de enero de 2016).

${ }^{16}$ Así, por ejemplo, Antxon Olabe se muestra categórico al señalar que "el acuerdo entre Pekín y Washington ha sido finalmente posible porque en los últimos años se ha instalado en EE UU la convicción de que el cambio climático supone una amenaza a la seguridad nacional", ver El camino hacia Paris, "Política Exterior" 163 (2015), disponible en:http://www.politicaexterior.com/articulos/ politica-exterior/el-camino-hacia-la-cumbre-de-paris/?added-to-cart=25868.

El texto de la Estrategia Nacional de Seguridad de 2015 parece afianzar este planteamiento, ya que se destacan los esfuerzos realizados en el país para el control de las emisiones como una contribución a la capacidad de liderazgo en la escena internacional, al tiempo que se anunciaba la colaboración con China para enfrentar el desafío y la amenaza que supone el cambio climático. Ver op. cit., p. 12. 


\section{El cambio climático desde la perspectiva de la seguridad internacional}

El proceso de securitización del cambio climático que se ha analizado en el apartado anterior respecto a los sistemas nacionales, conlleva en el ámbito internacional la percepción de que el protagonismo de su gestión también debe derivarse a aquellos órganos que tienen las principales competencias en el sistema de seguridad colectiva de Naciones Unidas, y en particular hacia el Consejo de Seguridad. Parece claro que incluir el cambio climático en la agenda de seguridad internacional hace que se convierta en una cuestión a tratar dentro de las competencias de los órganos que tienen que velar por la paz y la seguridad internacionales.

Se ha señalado al Reino Unido como el Estado pionero en el proceso de conexión del cambio climático con el concepto de seguridad nacional, pero debe recordarse además que es el que inicia el llamamiento para que el Consejo de Seguridad de las Naciones Unidas se ocupase de este riesgo ambiental, poniendo así de manifiesto la interconexión interna e internacional de las derivadas del proceso de securitización del cambio climático ${ }^{17}$. No obstante y pese a sucesivos llamamientos para que el Consejo de Seguridad desplegara una mayor actividad en materia de cambio climático, su labor y lade los principales órganos del sistema de las Naciones Unidas en este ámbito ha sido limitada, de forma que puede afirmarse que el proceso de securitización del cambio climático desde la perspectiva de la seguridad internacional se presenta con unos caracteres restringidos.

Si la pregunta clave en el ámbito de la seguridad nacional era cómo podía influir el proceso de securitización en la conformación de la posición internacional del Estado, en el ámbito de la seguridad internacional la principal cuestión es el papel que el Consejo de Seguridad debería desplegar en la gobernanza global del cambio climático. De nuevo en este escenario subyace la posibilidad de que destacar las implicaciones para la seguridad del cambio climático suponga una vía para superar la reticencia de los Estados a asumir compromisos climáticos ambiciosos. El Consejo de Seguridad está habilitado para declarar al cambio climático como una amenaza a la paz conforme al artículo 39 de la Carta de las Naciones Unidas, lo que supondría la viabilidad de activar sus competencias en virtud del Capítulo VIII de la Carta y, por tanto, de adoptar medidas obligatorias en este ámbito. Esta posibilidad resulta tentadora desde un punto de vista ambiental, ya que abre la capacidad de adoptar medidas vinculantes para combatir el cambio climático al margen de la difícil y compleja negociación internacional. No obstante, esta tentación no puede ocultar los problemas jurídicos, políticos y técnicos que presentarían una

\footnotetext{
${ }_{17}$ Ver Carta de 5 de abril de 2007 del Representante Permanente del Reino Unido e Irlanda del Norte en Naciones Unidas dirigida al Presidente del Consejo de Seguridad (S/2007/186).
} 
decisión y posterior actuación del Consejo de Seguridad en materia climática. Esta situación explica que este órgano no haya adoptado ninguna decisión concreta en este ámbito, aunque también que no pueda considerarse inactivo en esta materia ya que el cambio climático se incluyó en su agenda en 2007.

Como ya se ha apuntado, fue la iniciativa británica la que motivó el primer debate sobre cambio climático en el seno del Consejo de Seguridad, desarrollado en abierto el 17 de abril de $2007^{18}$. Esta primera reunión contó con una amplia participación, pero pudo observarse que no existía un consenso claro respecto a que la conexión entre cambio climático y seguridad pudiera motivar que este fenómeno ambiental se tratara en el marco de las competencias del Consejo de Seguridad. Los Estados en vías de desarrollo, entre los que destacaba la posición de China y la excepción de los Estados islas, mostraron sus dudas respecto a que se estuviera en el foro apropiado para abordar una materia cuyas principales implicaciones eran ambientales y de desarrollo ${ }^{19}$. Esta controversia explica que si bien fue posible que se afirmara en el debate la conexión entre cambio climático y seguridad internacional, no lo fuera la adopción posterior de ninguna decisión concreta. Del desarrollo del debate, puede extraerse una conclusión general, como es la conceptualización del cambio climático no como una amenaza autónoma, sino como un fenómeno ambiental que incide en la multiplicación e incremento de la gravedad de otras amenazas preexistentes. El cambio climático se configuraría así como una preocupación para la seguridad internacional, pero difícilmente podría motivar de forma autónoma una calificación de amenaza conforme al artículo 39 de la Carta.

Tras este primer debate en el Consejo de Seguridad, serían la Asamblea General y el Secretario General los que se harían eco de las repercusiones del cambio climático en materia de seguridad. El 3 de junio de 2009, la Asamblea General aprobó la Resolución 63/281, en la que por vez primera se reconocía la interconexión entre cambio climático y seguridad en un instrumento de Naciones Unidas ${ }^{20}$. Se pretendía iniciar así una línea de trabajo en la que se invitaba a participar al resto de órganos de las Naciones Unidas, y se incluía al Secretario General mediante la petición de un informe sobre las posibles repercusiones del cambio climático para la seguridad. Este llamamiento sería la causa del informe que el Secretario General presentó a la Asamblea General de las Naciones Unidas en su $64^{\circ}$ periodo de sesiones, con el título de $E l$

\footnotetext{
${ }^{18}$ Ver Security Council Holds First-ever Debate on Impact of Climate Change on Peace (SC/9000, 17 April 2007).

${ }^{19}$ En particular, China declaró de forma contundente que: "the developing countries believe that the Security Council has neither the professional competence in handling climate change -- nor is it the right decision-making place for extensive participation leading up to widely acceptable proposals". Ibidem.

${ }^{20}$ Ver El cambio climático y sus posibles repercusiones para la seguridad, A/RES/63/281, 11 de junio de 2009.
} 
Cambio climático y sus posibles implicaciones en materia de seguridad ${ }^{21}$. En este documento el cambio climático sería calificado como un multiplicador de amenazas, señalando que es un factor que puede incrementar las fuentes existentes de conflicto e inseguridad ${ }^{22}$. La actividad de la Asamblea General y del Secretario General diseñaban de esta forma la conceptualización del cambio climático como un factor multiplicador de riesgos y amenazas para la seguridad, reconociendo tajantemente las implicaciones para la seguridad de este fenómeno ambiental, pero sin calificarlo de amenaza autónoma. El cambio climático entraba así definitivamente en la agenda de seguridad de las Naciones Unidas.

El Consejo de Seguridad volvería a tratar la cuestión del cambio climático en un nuevo debate abierto el 20 de julio de $2011^{23}$. Esta sesión se desarrolló bajo la presidencia alemana con un claro liderazgo europeo, y la preocupación por la situación de los pequeños Estados islas en vías de desarrollo protagonizó buena parte de las intervenciones. La aproximación al cambio climático como un factor multiplicador de amenazas fue reiterada en este encuentro, en el que se insistió en el planteamiento de la cuestión que habían desarrollado la Asamblea y el Secretario General. Poco más se aportó respecto a esta conceptualización, pero lo que resultó interesante de aquella reunión es que se estaba abordando con especial interés uno de los problemas en los que se muestra con claridad la conexión entre cambio climático y seguridad, como es el de la situación de los pequeños Estados islas en vías de desarrollo en relación a la subida del nivel del mar. El Consejo de Seguridad se situaba así ante un asunto concreto que ha quedado ya incluido entre sus preocupaciones, y que requerirá una especial atención en la que tendrá que abordarse la reflexión sobre cómo actuar sobre ese cambio climático que se presenta como un multiplicador de los riesgos y amenazas que enfrentan estos Estados ${ }^{24}$.

Los debates oficiales en el seno del Consejo de Seguridad específicos sobre cambio climático se han limitado a las dos sesiones citadas, pero merece

\footnotetext{
${ }^{21}$ Ver El Cambio climático y sus posibles implicaciones en materia de seguridad, A/64/350, $11 \mathrm{de}$ septiembre de 2009.

${ }^{22}$ Ver Ibidem, par. 13.

${ }^{23}$ Ver Statement by the President of the Security Council, 20 July 2011 (S/PRST/2011/15).

${ }^{24}$ El Consejo de Seguridad se ha ocupado recientemente de la situación de los pequeños Estados islas en vías de desarrollo en un debate abierto celebrado el 30 de julio de 2015. En este encuentro se calificó al cambio climático como un multiplicador de los riesgos y amenazas que enfrentaban estos Estados, enfatizándose el interés de avanzar en su mitigación y adaptación para la seguridad de sus territorios y poblaciones. Ver Peace and Security Challenges Facing Small Island Developing States, 30 July 2015 (SC/11991).

Sobre la cuestión particular de la situación de los pequeños Estados islas en vías de desarrollo y la incidencia del cambio climático en materia de seguridad, puede consultarse el trabajo de Mariano J. Aznar Gómez, El Estado sin territorio: la desaparición del territorio debido al cambio climático, "Revista Electrónica de Estudios Internacionales", 26 (2013), disponible en: http://www.reei.org/ index.php/revista/num26/articulos/estado-sin-territorio-desaparicion-territorio-debido-al-cambioclimatico.
} 
la pena dedicar unas líneas a las reuniones abiertas que utilizando la Fórmula Arria se realizaron sobre esta cuestión el 15 de febrero de 2003, y el 30 de junio de $2015^{25}$. Los Estados convocantes del primer encuentro oficioso del Consejo de Seguridad bajo esta fórmula fueron Reino Unido y Pakistán, mientras que el segundo fue auspiciado por España y Malasia. Ambos encuentros se enmarcaron en la voluntad de mantener abierta también esta vía de discusión, que por su carácter flexible puede aportar resultados interesantes a la hora de abordar el cambio climático desde la óptica de la seguridad. En la segunda reunión se aprecia además el interés por avanzar en un dialogo que también podía ser provechoso para que se alcanzaran resultados relevantes en la reunión de la Conferencia de París del mismo año. No es casualidad la proximidad de la fecha, y este encuentro muestra, de nuevo, que la voluntad de avanzar en el enfrentamiento del cambio climático como un problema de seguridad se muestra como paralelo al desarrollo de compromisos en el régimen internacional en materia de clima. Es previsible que esta fórmula siga utilizándose en el Consejo de Seguridad para abordar la cuestión del cambio climático en los próximos años, si es así, habrá que prestar atención a este nuevo foro de diálogo y a su posible interconexión con la negociación climática global.

Si se reflexiona sobre la labor del Consejo de Seguridad que ha sido enunciada, aparece con claridad la percepción de que este órgano ha reconocido que el cambio climático tiene importantes implicaciones para la seguridad, no obstante, poco más se puede decir respecto a cómo conceptualizaría a este fenómeno desde la óptica de la seguridad y qué medidas podría adoptar al respecto. Resulta evidente que una posible calificación del cambio climático como amenaza a la paz en el sentido del artículo 39 de la Carta está muy lejos de poder producirse. La composición misma del Consejo de Seguridad podría explicar esta postura, en su seno y como miembros permanentes se observan a grandes emisores de gases de efecto invernadero, pero además los Estados en vías de desarrollo han mostrado reiteradamente en los debates celebrados sus dudas de que sea el foro adecuado para esta cuestión ${ }^{26}$.

En particular, China se ha mostrado reticente a considerar la cuestión del cambio climático como un problema de seguridad desde la primera reunión celebrada en 2007, enfatizando que la aproximación apropiada sería la que

${ }^{25}$ El primer encuentro se dearrolló bajo el título de Arria Formula Meeting onThe Security Dimensions of Climate Change, 15 February 2003, y no fue emitida documentación al respecto. Como resultado del segundo puede consultarse la Concept Note: Open Arria-formula Meeting onthe Role of Climate Change as a Threat Multiplierfor Global Security, 30 June, 2015, emitida por los Gobiernos de España y Malasia, disponible en: http:/www.spainun.org/wp-content/uploads/2015/06/ConceptNote_ClimateChange_20150630.pdf (última visita el 15 de enero de 2016).

${ }^{26}$ Sobre la posición de los Estados en vías de desarrollo en la actividad del Consejo de Seguridad, ver Shirley V. Scott, The Securitization of Climate Change in World Politics: How Close Have We Come and Would Full Securitization Enhance the Efficacy of Global Climate Change Policy?, "Review of European Community \& International Environmental Law, (21) 2012, pp. 220-230, p. 225 . 
tiene que ver con el desarrollo sostenible, y por lo tanto con la aplicación de principios diseñados en un contexto diferente al de la seguridad como el principio de responsabilidades comunes pero diferenciadas de los Estados. Puede afirmarse, por tanto, que en este contexto se ha producido una importante reticencia de los países en desarrollo a aceptar un proceso de securitización del cambio climático, debido a que podría trasladar la toma de decisiones a ámbitos diferentes al del régimen en materia de cambio climático, en el que se aceptan los principios de interconexión entre protección ambiental y desarrollo a través del paradigma del desarrollo sostenible.

Ahora bien, aunque pudieran superarse las reticencias mostradas en el Consejo de Seguridad para caracterizar al cambio climático como una amenaza a la paz, pervivirían problemas a la hora del diseño de las medidas que deberían adoptarse para abordarla. El fenómeno del cambio climático presenta una importante complejidad científico-técnica, en la que en la que es un elemento definitorio la pervivencia de la incertidumbre sobre algunos aspectos de sus causas y consecuencias, y la valoración de las medidas que deberían adoptarse para combatirlo ${ }^{27}$. Ante esta complejidad e indefinición el Consejo de Seguridad no aparece como el mejor dotado para poder diseñar estrategias con las que enfrentar este desafío ${ }^{28}$. En su reunión de 2011, el propio Consejo de Seguridad reconoce que necesita información para poder abordar el tema, y le pide al Secretario General que se la proporcione. La cuestión es que para obtener esta información y recibir la ayuda necesaria para diseñar medidas eficaces, tendrá que recabar la asistencia de los órganos del régimen internacional en materia de cambio climático, único sistema con un desarrollo suficiente en la materia para poder dárselo.

En todos los documentos emitidos tanto desde el Consejo de Seguridad como de la Asamblea o del Secretario General, se ha reconocido que el régimen internacional en materia de clima es el foro adecuado para enfrentar en primer término este fenómeno ambiental ${ }^{29}$. Ha sido también reiterado el llamamiento

\footnotetext{
${ }^{27}$ El Secretario General de Naciones Unidas afirmó que “[...] si bien la elaboración de modelos climáticos ha hecho avances considerables en la predicción del comportamiento futuro de los sistemas naturales a lo largo de periodos prolongados, la ciencia del cambio climático y sus efectos físicos sigue estando sujeta a algunas incertidumbres, por ejemplo en cuanto a la magnitud, el alcance geográfico y la escala temporal de determinados efectos”, op. cit., par. 8.

28 José Juste Ruíz describe con precisión la circunstancia a la que se tendría que enfrentar al señalar que "el carácter global de los problemas de seguridad producidos por el cambio climático viene marcado por dos rasgos de particular importancia. Por una parte, se trata de un fenómeno que tiene orígenes difusos, ya que las fuentes de emisión de los gases de efecto invernadero que están en el origen del problema se sitúan en todas partes. De otro lado, los efectos presumibles del cambio climático sobre el medio ambiente son generalizados pero aleatorios, siendo imposible determinar a priori con certeza cuáles serán los países particularmente afectados por los efectos adversos de este fenómeno". Ver La seguridad ambiental transfronteriza: el marco jurídico internacional [en Pablo Antonio Fernández Sánchez y José Alberto Azeredo Lopes, dirs.: Seguridad medioambiental y cooperación transfronteriza, Atelier, 2015], pp. 13-34.

29 Para una mayor reflexión sobre esta aproximación, ver Shirley V. Scott, op. cit.
} 
a que fuera apoyado el desarrollo de compromisos en el marco del régimen climático. Ante las dificultades del Consejo de Seguridad para poder enfrentar el desafío del cambio climático, y la observación de estas declaraciones, puede concluirse que la vía que puede resultar interesante explorar es la de una mayor colaboración y apoyo de los órganos de seguridad del sistema de Naciones Unidas y el régimen de clima. Esta interconexión está lejos de producirse, pero quizás la línea de debates formales e informales del Consejo de Seguridad pueda favorecer su consecución. El dialogo que podría establecerse puede incidir en un doble plano: impulsar la adopción de compromisos climáticos como derivada de un proceso, aunque sea incompleto, de securitización internacional; y favorecer la adopción de medidas con una aproximación climática en la labor del Consejo de Seguridad, en particular durante el desarrollo de las Operaciones de Mantenimiento de la Paz.

\section{El cambio climático desde la perspectiva de la seguridad humana}

En la década de los noventa del pasado siglo se articuló una nueva aproximación al concepto de seguridad que daría como resultado la adopción del térmico seguridad humana, introducido por vez primera en el informe sobre el desarrollo humano de 1994, Un programa para la Cumbre Mundial sobre Desarrollo Social, del Programa de Naciones Unidas para el Desarrollo (PNUD). Con este concepto se pretendía configurar un concepto integral y extensivo, en el que la seguridad fuera más allá de la mera preocupación por el conflicto armado, para incluir la preocupación por la vida y la dignidad humana. En el informe de 1994 se señalaba que esta nueva dimensión de la seguridad se caracterizaba por la universalidad, la interdependencia de sus componentes, su centralidad sobre las personas, y que quedaba mejor garantizada mediante la prevención. Conforme a esta descripción, debía incluir la seguridad económica, la seguridad alimentaria, la seguridad en materia de salud, la seguridad ambiental, la seguridad personal y la seguridad de la comunidad política, utilizando una relación en la que se pretendía incluir las principales áreas en las que incidirían las diversas amenazas que pueden desarrollarse. De esta forma se trataba de superar el concepto tradicional basado en la preocupación por la integridad del Estado, y situar la protección de individuos y colectividades en el núcleo del concepto ${ }^{30}$.

${ }^{30}$ El Grupo de Alto Nivel sobre las Amenazas, los Desafíos y el Cambio, señalaba en diciembre de 2004 lo siguiente respecto de la idea de seguridad, muy oportuno en relación con el tema que nos toca tratar: "Cualquier suceso o proceso que cause muertes en gran escala o una reducción masiva en las oportunidades de vida y que socave el papel del Estado como unidad básica del sistema internacional constituye una amenaza a la seguridad internacional. Si se parte de esa definición, hay seis grupos de amenazas que deben preocupar al mundo hoy y en los decenios por venir: las amenazas económicas y sociales, como la pobreza, las enfermedades; infecciosas y la degradación 
Desde que el término fue acuñado en la práctica internacional, ha suscitado gran interés tanto en el debate internacional como en la reflexión académica. Pese a esta popularidad, el concepto sigue resultando algo difuso y es difícil delimitar sus elementos particulares. No puede perderse de vista que esta noción emana desde el ámbito del Derecho Internacional al Desarrollo, por lo que en buena medida va a compartir algunos de los rasgos de este sector jurídico internacional en el que las normas de softlaw ocupan un amplio espacio. No cabe duda además de que se conecta con un proceso de securitización, ya que pretende trasladar al campo de la seguridad el análisis de múltiples situaciones en las que se vulneran derechos fundamentales de individuos y colectividades. Estos elementos confluyen para conformar un concepto de difícil aprehensión práctica, lo que ha suscitado no pocas críticas tanto por la dificultad de su implementación, como por incluir planteamientos contradictorios respecto al respeto de la dignidad de los colectivos protegidos ${ }^{31}$.

Al margen de la amplia controversia suscitada por este concepto, interesa a este estudio incluir algunas reflexiones sobre lo que podría aportar en su conexión con el cambio climático. Resulta claro que los impactos del cambio climático pueden suponer una clara amenaza para la seguridad humana, configurándose como uno de los riesgos por excelencia de la supervivencia del ser humano en el planeta. Por un lado, el cambio climático constituye en sí mismo una amenaza desde la óptica de este concepto de seguridad; pero además potenciaría prácticamente la totalidad de los riesgos que pudieran incluirse en cualquier listado, actuando plenamente como un multiplicador de amenazas. Ahora bien, aunque el impacto del cambio climático será global, no cabe duda de que su incidencia será mayor en los países con un menor desarrollo, en los que las capacidades de adaptación son muy escasas.

Ante la situación descrita, la interrelación entre cambio climático y el concepto de seguridad humana lleva a destacar los elementos que tienen que ver con el dispar desarrollo de los Estados, y con la necesidad de los menos desarrollados a recibir ayuda para enfrentar este fenómeno ambiental. Se ha señalado en el apartado anterior que los Estados en vías de desarrollo se han mostrado reacios a vincular la cuestión del cambio

ambiental; los conflictos entre Estados; los conflictos internos, como la guerra civil, el genocidio y otras atrocidades en gran escala; las armas nucleares, radiológicas, químicas y biológicas; el terrorismo; la delincuencia organizada transnacional". Ver el Informe Un mundo más seguro: la responsabilidad que compartimos, A/59/565, de 2 de diciembre de 2004, p. 24.

${ }^{31}$ Así, por ejemplo, Elena Conde ha señalado que "desde el punto de vista de quien escribe estas líneas, la denominada 'seguridad humana' es un concepto a la moda de la securitización generalizada para referirse de forma atractiva a algo ya existente que, efectivamente, tiene que ver con la idea de solidaridad y que, en otros ámbitos, como el derecho del medio ambiente, se llamaría equidad intergeneracional o, incluso, sostenibilidad”, El impacto del cambio climático en la seguridad y la defensa: Introducción, [en Ministerio de Defensa, Energía y Clima en el área de la seguridad y la defensa, Ministerio de Defensa, 2013], pp. 82-92, p. 91.

El Grupo de Alto Nivel sobre las Amenazas, los Desafíos y el Cambio sería contundente al afirmar en su informe de 2004 que: "si bien las Naciones Unidas dieron origen al concepto de la seguridad humana, demostraron estar mal preparadas para proporcionarla”, op. cit., p. 20.

Araucaria. Revista Iberoamericana de Filosofía, Política y Humanidades, año 18, n 36. Segundo semestre de 2016. Pp. 315-338. ISSN 1575-6823 e-ISSN 2340-2199 doi: 10.12795/araucaria.2016.i36.14 
climático y el concepto de amenaza a la seguridad, ante el temor de que el protagonismo de la esfera basada en la seguridad desplazara a una negociación ambiental en la que tradicionalmente se ha reivindicado el principio de responsabilidades comunes pero diferenciadas de los Estados ${ }^{32}$. El concepto de seguridad humana supone una noción más fácil de aceptar para estos Estados, ya que en sí misma incorpora los rasgos de una reivindicación del respeto al derecho al desarrollo, y de una necesidad de ayuda adicional para cumplir compromisos ambientales. Si se reflexiona sobre los elementos que aportaría al régimen sobre cambio climático una aproximación basada en el concepto de seguridad humana, aparece la similitud con las reivindicaciones que tradicionalmente han realizado los Estados en vías de desarrollo.

Para la protección de individuos y comunidades, el concepto de seguridad humana llevaría a promover la adopción de una acción preventiva respecto al cambio climático que conllevara la adopción de medidas de mitigación, al tiempo que una acción reactiva con medidas de adaptación. No puede olvidarse que el concepto de seguridad humana surge como defensa de bienestar del ser humano, pero en el marco del ámbito del desarrollo, por lo que aparece como un parámetro que afianza la necesidad urgente de cumplir los dos objetivos del régimen de cambio climático. Al poner el foco en el bienestar humano y señalar la diferente vulnerabilidad frente a las consecuencias del cambio climático, este concepto apunta, precisamente, a la conveniencia de que mitigación y adaptación sean objetivos paralelos, en el que el segundo no pierda protagonismo frente al primero a pesar de su menor coherencia ambiental. La reivindicación de los Estados en vías de desarrollo más vulnerables ha sido precisamente ésta, y parece haber alcanzado cierta respuesta al incluirse las medidas de adaptación en el contenido de las INDCs conforme al nuevo Acuerdo de París ${ }^{33}$.

El encuadre de la seguridad humana en un contexto general de desarrollo también trae aparejada la necesidad de que se potencia una financiación del cambio climático en el que los Estados en vías de desarrollo reciban asistencia adicional por la implementación de compromisos ambientales. El actual sistema de financiación incluye tanto las medidas dirigidas a la mitigación como a la adaptación, de forma que, de nuevo, en el primer aspecto tiene un carácter preventivo, mientras que en el segundo es paliativo y entra en conexión directamente con casos en los que el riesgo se ha transformado en realidad. La financiación se ha mostrado como uno de los aspectos más controvertidos de la negociación climática, y ha sido señalado como indispensable para la participación de los Estados en vías de desarrollo en cualquier sistema de obligaciones climáticas. El nuevo Acuerdo de París incide en un camino ya encauzado a través del mecanismo financiero de la CMNUCC, y que supone el reconocimiento de los Estados desarrollados de este elemento clave para promover una aceptación global de compromisos climáticos que incluya a los Estados en vías de desarrollo.

\footnotetext{
${ }^{32}$ Ver el reconocimiento del principio de responsabilidades comunes pero diferenciadas en el Principio 7 de la Declaración sobre Desarrollo Sostenible de Río de Janeiro; y el artículo 3.1 CMNUCC.

${ }^{33}$ Ver art. 4 y siguientes del Acuerdo de París, Decisión 1/CP. 21.
} 


\section{Conclusiones}

El gran desafío del régimen internacional en materia de cambio climático es materializar el compromiso de los Estados Partes para desarrollar acciones altamente costosas frente a un fenómeno ambiental en el que persisten importantes incertidumbres. Ante esta situación, se plantea de forma general que el proceso de securitización puede suponer la ventaja de añadir un impulso que permita avanzar en la negociación. Cuando se declara que el cambio climático es una amenaza para la seguridad, pasa de enmarcarse en el Derecho Internacional del Medio Ambiente, un ámbito muchas veces criticado por tener una escasa capacidad de abordar de forma contundente los desafíos planteados, al Derecho Internacional de la paz y la seguridad, en el que la capacidad para imponer acciones y sanciones resulta factible. En materia climática, este proceso puede conllevar el efecto de hacer tangible y sólido un peligro frente al que los Estados estén dispuestos a actuar, y esto puede revertir en efectos positivos y de avance del régimen. Al fin y al cabo, el miedo siempre se ha mostrado como el gran aliado de la acción ambiental.

Esta reflexión parece correcta en lo que respecta a aquellos sistemas nacionales en los que se ha incluido al cambio climático en la agenda de seguridad. En los Estados desarrollados ha sido habitual desplegar un proceso en el que se asumían los efectos sobre la seguridad del cambio climático, y se generaba la percepción de que se convertía en un problema que habría que gestionar desde esta óptica. Este proceso ha facilitado, en estos Estados, la configuración de una posición en el régimen internacional en materia de clima más proclive a asumir compromisos. El caso de Estados Unidos es especialmente relevante en este ámbito, convirtiéndose el proceso de securitización en un elemento importante con el que enfrentar las reticencias internas a asumir obligaciones climáticas ambiciosas, y ejercer el liderazgo en este escenario. Los procesos de securitización pueden resultar, por tanto, favorecedores para la mayor implicación de los Estados desarrollados en el avance del régimen en materia de cambio climático.

Cuando la observación se sitúa en la óptica de la seguridad internacional, el proceso de securitización no parece tan concluyente. La introducción del cambio climático en la agenda de los principales órganos implicados en el sistema de seguridad colectiva de las Naciones Unidas, en particular en la del Consejo de Seguridad, muestra el reconocimiento de que este fenómeno puede incidir o agravar diversos riesgos y amenazas. No obstante, no existe el consenso necesario para poder prever que el Consejo de Seguridad pueda avanzar en la conceptualización del cambio climático como una amenaza a la paz, y en todo caso no parece que se configure como el órgano más capacitado para el diseño de soluciones eficaces en este ámbito. En este caso, el proceso 
de securitización podría conllevar que se desarrollara un mayor respaldo al régimen de cambio climático, al constituirse el Consejo en un foro adicional de negociación en el que desarrollar la confianza mutua entre los Estados que tan necesaria se muestra en la negociación climática.

Los Estados en vías de desarrollo se han mostrado reacios a que el cambio climático se abordarse en el Consejo de Seguridad, y han expresado sus dudas sobre si la aproximación desde la seguridad era la más adecuada a este problema. Para estos Estados el cambio climático debe abordarse prioritariamente con una aproximación basada en el desarrollo, de forma que se aprecie la necesidad de recibir ayuda de estos Estados para asumir compromisos ambientales. La oposición a un proceso de securitización climático se atenúa cuando se introduce una noción de seguridad más omnicomprensiva, que conecta con buena parte de los principios enunciados en el ámbito del desarrollo. La noción de seguridad humana aporta una aproximación a la interconexión entre cambio climático y seguridad a la que los Estados en vías de desarrollo son más favorables. Desde esta óptica, la preocupación por la seguridad aporta derivadas parecidas a las que se realizarían desde la aplicación del principio de responsabilidades comunes pero diferenciadas de los Estados, enfatizando la necesidad de desarrollar medidas de adaptación y de financiación en el régimen internacional en materia de clima.

Las diferentes aproximaciones al concepto de seguridad que se han utilizado en este trabajo han permitido desarrollar diferentes reflexiones en torno al impacto de la securitización en el desarrollo del régimen internacional para el cambio climático. Mientras que el análisis desde la seguridad nacional e internacional permite señalar cómo este proceso puede favorecer la búsqueda de la aceptación de compromisos climáticos, el realizado desde la seguridad humana ahonda en la constatación de que hay que afrontar la reivindicación de los Estados en vías de desarrollo de diseñar una acción ambiciosa desde un punto de vista ambiental, pero también de desarrollo. La cuestión no puede más que quedar abierta, en una fase transitoria de un régimen internacional en materia de clima que abre nuevas posibilidades tras la adopción del Acuerdo de París. 


\section{Referencias bibliográficas:}

Abad Quintanal, Gracia, El concepto de seguridad: su transformación, "Comillas Journal of International Relations", 4 (2015), pp. 41-51.

Aznar Gómez, Mariano J., El Estado sin territorio: la desaparición del territorio debido al cambio climático, "Revista Electrónica de Estudios Internacionales", 26 (2013), disponible en: http://www.reei.org/index. $\mathrm{php} /$ revista/num26/articulos/estado-sin-territorio-desaparicion-territoriodebido-al-cambio-climatico

Balzacq, Thierry, Securitization Theory: How Security Problems Emerge and Dissolve, Routledge, 2010.

Brauch, Günter; Link, Peter Michael; y Schilling, Janpeter, editores, Climate Change, Human Security and Violent Conflict. Challenges for Societal Stability, Springer, 2012.

Buzan, Barry; Wæver, Ole; y Wilde, Jaap de, Security. A New Framework for Analysis, Lynne Rienner Publishers, 1998.

Conde, Elena, El impacto del cambio climático en la seguridad y la defensa: Introducción, [en Ministerio de Defensa, Energía y Clima en el área de la seguridad y la defensa, Ministerio de Defensa, 2013], pp. 82-92.

García Sánchez, Ignacio José, La Estrategia de Seguridad Nacional de los Estados Unidos de América 2015, "Documento de Análisis. Instituto Español de Estudios Estratégicos" 9 (2015), pp. 1-9.

Giles Carnero, Rosa, El Protocolo de Kioto como modelo de gestión ambiental global, en [Antonio Remiro Brotòns y Rosa Ma Fernández Egea, eds., El Cambio Climático en el Derecho Internacional y Comunitario, Fundación BBVA, 2009], pp. 27-601.

Giles Carnero, Rosa, La acción internacional y europea en materia de cambio climático: construyendo la nueva fase 2013-2020 [en Isabel Rodríguez Martínez, directora, La negociación de emisiones GEI en los mercados de carbono. Régimen y regulación, Thomson Reuters Aranzadi, 2014], pp. 39-62.

Harris, Katie, Climate Change in UK Security Policy: Implications for development Assistance?, Working Paper 342. Overseas Development Institute, 2012.

Juste Ruíz, José, La seguridad ambiental transfronteriza: el marco jurídico internacional [en Pablo Antonio Fernández Sánchez y José Alberto Azeredo Lopes, dirs.: Seguridad medioambiental y cooperación transfronteriza, Atelier, 2015], pp. 13-34. 
Olabe, Antxon, El camino hacia París, "Política Exterior" 163 (2015), disponible en: http:/www.politicaexterior.com/articulos/politica-exterior/ el-camino-hacia-la-cumbre-de-paris/?added-to-cart=25868.

Scott, Shirley V., The Securitization of Climate Change in World Politics: How Close Have We Come and Would Full Securitization Enhance the Efficacy of Global Climate Change Policy?, "Review of European Community \& International Environmental Law", (21) 2012, pp. 220-230, p. 225.

Torres Cazorla, María Isabel y García Rico, Elena del Mar, coordinadoras, La seguridad internacional en el siglo XXI: nuevas perspectivas, Plaza y Valdés Editores, 2011.

Wilkinson, Claire, The Copenhagen School on Tour in Kyrgyzstan: Is Securitization Theory Useable Outside Europe?, "Security Dialogue", (38) 2007, pp. 5-25. 